University of Wollongong

Research Online

Faculty of Engineering and Information

Faculty of Engineering and Information

Sciences - Papers: Part A

Sciences

January 2006

\title{
Monte Carlo simulation of electromagnetic interactions of radiation with liquid water in the framework of the Geant4 DNA project
}

\author{
S Chauvie \\ INFN Sezione di Genova, Italy \\ Z Francis \\ S Guatelli \\ University of Wollongong, susanna@uow.edu.au \\ S Incerti \\ University Bordeaux \\ B Mascialino \\ Karolinska Institute, barbara.mascialino@ki.se
}

See next page for additional authors

Follow this and additional works at: https://ro.uow.edu.au/eispapers

Research Online is the open access institutional repository for the University of Wollongong. For further information contact the UOW Library: research-pubs@uow.edu.au 


\title{
Monte Carlo simulation of electromagnetic interactions of radiation with liquid water in the framework of the Geant4 DNA project
}

\author{
Abstract \\ A set of processes specialized for the simulation of particle interactions with water has been developed in \\ the Geant4 Low Energy Electromagnetic package. The models cover the interactions of electrons, protons \\ and light ions down to the electronvolt energy scale. They address a physics domain relevant to the \\ simulation of radiation effects in biological systems, where water represents an important component. \\ The software design, the physics models implemented and an overview of their tests are described.

\section{Keywords} \\ framework, electromagnetic, liquid, radiation, project, water, dna, interactions, geant4, monte, carlo, \\ simulation

\section{Publication Details} \\ Chauvie, S., Francis, Z., Guatelli, S., Incerti, S., Mascialino, B., Montarou, G., Nieminen, P. \& Pia, M. G. \\ (2006). Monte Carlo simulation of electromagnetic interactions of radiation with liquid water in the \\ framework of the Geant4 DNA project. In B. Phlips (Eds.), IEEE Nuclear Science Symposium Conference \\ Record (pp. 796-802). New Jersey, United States: IEEE.

\section{Authors} \\ S Chauvie, Z Francis, S Guatelli, S Incerti, B Mascialino, G Montarou, P Nieminen, and M G. Pia
}




\title{
Monte Carlo Simulation of Electromagnetic Interactions of Radiation with Liquid Water in the Framework of the Geant4-DNA Project
}

\author{
S. Chauvie, Z. Francis, S. Guatelli, S. Incerti, B. Mascialino, G. Montarou, P. Moretto, P. Nieminen, \\ M. G. Pia
}

\begin{abstract}
A set of processes specialized for the simulation of particle interactions with water has been developed in the Geant4 Low Energy Electromagnetic package. The models cover the interactions of electrons, protons and light ions down to the electronVolt energy scale. They address a physics domain relevant to the simulation of radiation effects in biological systems, where water represents an important component. The software design, the physics models implemented and an overview of their tests are described.
\end{abstract}

\section{INTRODUCTION}

$\mathrm{T}$ HE simulation of radiation effects in biological systems is a critical concern in various domains, such as oncological radiotherapy and radiation protection. The irradiation of a biological system is usually described in terms of the dose released to it; nevertheless the concept of dose is not adequate to estimate the radiation effects when microscopic entities, such as cellular structures and the DNA (deoxyribonucleic acid) molecule, are the object of study.

The Geant4-DNA project addresses the extension of the Geant4 [1]-[2] simulation toolkit to deal with radiation effects at the cellular and DNA scale. It exploits the Geant4 architectural design to equip an open source, general purpose Monte Carlo system for particle transport for the first time with functionality for simulation specific to radiobiology applications. This novel approach allows scientists to exploit

Manuscript received November 27, 2006. Work partly supported through ESA/ESTEC Contract No. 14402/00/NL/MV.

S. Chauvie is with Santa Croce e Carle Hospital, Cuneo and with INFN Sezione di Torino, I-10125 Torino, Italy (e-mail: chauvie@to.infn.it).

Z. Francis and G. Montarou are with Université Blaise Pascal, LPC, IN2P3/CNRS, F-63177 Aubière Cedex, France (e-mail: francis@clermont. in2p3.fr, montarou@clermont.in2p3.fr)

S. Incerti and P. Moretto are with CENBG - Centre d'Etudes Nucléaires de Bordeaux-Gradignan, IN2P3/CNRS, Bordeaux 1 University, F-33175 Gradignan Cedex, France (e-mail: incerti@cenbg.in2p3.fr, moretto@cenbg. in2p3.fr)

S. Guatelli, B. Mascialino and M. G. Pia are with INFN Sezione di Genova, Via Dodecaneso 33, I-16146 Genova, Italy (e-mail: Barbara.Mascialino@ge. infn.it, MariaGrazia.Pia@ge.infn.it)

P. Nieminen is with ESA/ESTEC, Postbus 299, 2200 AG Noordwijk, The Netherlands (e-mail: Petteri.Nieminen@esa.int). specialized tools for the study of the biological effects of radiation together with the rich simulation functionality of a full Monte Carlo system for particle transport; nevertheless, thanks to the toolkit structure of Geant4, no unnecessary overheads are introduced for using a general purpose simulation system rather than a Monte Carlo code specialized for radiobiology simulation: in fact, a Geant4 application only uses the toolkit components needed to satisfy its functionality.

The Geant4-DNA software encompasses various domains: the extension of Geant 4 physics processes down to the energy scale relevant to bio-molecular systems, the development of models to describe biological processes, and the description of biological entities, such as cells or the DNA molecule. The overall Geant4-DNA subsystem adopts a component-based architecture, where each domain is addressed by a software component.

This paper describes a new component of Geant4 physics developed in the context of the Geant4-DNA project: a set of processes to model particle interactions in water, the main component of biological systems, down to the electronvolt energy scale. These new developments are identified in the following sections as the Geant4-DNA Physics Processes; they are part of the Geant4 Low Energy Electromagnetic Physics package [3]-[4]

The Geant4-DNA software is open-source; it is publicly released as part of the Geant4 Toolkit.

\section{SOFTWARE PROCESS}

The Geant4-DNA project addresses a very active scientific domain: the continuous progresses in biological research, as well as the availability of new accelerator facilities for cellular irradiation, contribute to a rapid evolution of the body of knowledge in the field. The development of the Geant4-DNA software follows an iterative and incremental process. Such a process model is particularly suitable to address the complex, fast evolving research domain of radiation effects on biological systems: it allows building and refining the software taking into account the theoretical and experimental progresses in the field, at the same time producing concrete deliverables at each development cycle.

The software process of Geant4-DNA is based on the 
Unified Software Development Process [5] and adopts the Rational Unified Process ${ }^{\mathrm{TM}}$ (RUP) [6] as a process framework, tailored to the specific characteristics of the project and its scientific environment [7]. The rigorous software process, supported by the assessment [8] of the RUP against the ISO 15504 [9] standard, is a key element contributing to the quality of the Geant4-DNA software. The main features of the software process are illustrated in the following sections.

The dynamic dimension of the software process is embedded in the more general lifecycle of Geant 4 . The first development cycle of the Geant4-DNA project investigated the complex problem domain and identified its most relevant requirements; the concrete software development at that early stage consisted of exploratory prototypes only. The following development cycles have been articulated across the regular public releases of Geant4; each evolution cycle addresses a specific sub-domain of the project, according to an iterative and incremental process.

This paper describes the software resulting from the second development cycle, which corresponds to a preliminary version of the Geant4-DNA physics processes in Geant4 8.1 and a refined version to be released in Geant 4 version 9.

\section{A. Requirements}

The high level requirements for the simulation of physics interactions relevant to radiobiology were identified and documented in the inception phase of the project. The second development cycle extended the functional requirements and identified a few essential non-functional ones.

The primary functional requirements for the simulation of radiation effects in biological systems consist in the capability to model the physics interactions of a variety of incident particles at least in water, which is the main component of biological tissue; the physics models must cover energies down to the electronVolt scale, to describe effects relevant to the size of cellular and sub-cellular systems. The software should be able to calculate the cross sections of all the processes involved, and to generate the secondary particles resulting from the interaction of the primary particles with the medium.

A few non-functional requirements play an important role in driving the Geant4-DNA software development. Flexibility to adapt to a rapidly evolving scientific environment is a key requirement of the Geant4-DNA software, as well as the need to accommodate different physics models. Performance optimization is also an important requirement: radiobiological simulations are computationally intensive, since they require tracking primary and secondary particles down to very low energies compatible with cellular and sub-cellular dimensions.

The requirements of Geant4-DNA are documented in a User and Software Requirements Document. The document is updated according to the evolution of the requirements. Use case models are extracted from the requirements to support the analysis and design process.

The requirements and associated use cases drive the software process of the project. They are subject to traceability throughout the whole development cycle; the traceability process links the requirements, the design, the code (with reference to the theoretical models it is based on), the unit tests and related physics verification results, the integration tests, the physics validation tests with their reference data and related results, and the user documentation.

\section{B. Analysis and design}

This discipline plays a key role in the whole development of the Geant4-DNA project, consistently with the architecturecentric characteristic of the software process model adopted. Sound software architecture is essential to cope with the complexity of the problem domain and the rapid evolution of the requirements in an active field of research.

The design of the Geant4-DNA physics processes derives from the analysis of the user and software requirements. The flexibility of modeling a complex physics domain and the concern for execution overheads drive the software architecture, while the functional requirements drive the detailed design of the software.

The design of the Geant4-DNA physics processes is described in detail in section III. It is the result of a few iterations, which refined the initial candidate architecture according to the iterative and incremental software process adopted. Round-trip engineering contributed to the design process: the feedback that candidate design solutions could be supported by commonly available compilers was essential to mitigate the risks of introducing innovative programming techniques in Geant4.

\section{Implementation}

The software implementation obeys to the general coding guidelines of Geant4.

The implementation discipline takes into account some specific features of the innovative design adopted for the Geant4-DNA physics processes. As described in section III, the design technique employed makes use of templates; the support of this coding language feature according to the $\mathrm{C}++$ standard is still under evolution in most compilers. Therefore, the implementation process comprises a frequent integration of the code on Geant4-supported platforms, to verify its compatibility with a variety of compilers at an early stage of the software development.

The code implementation interplays closely with the test process, which is described in detail in the following section.

\section{Testing}

The test process of the Geant4-DNA software complies with the more general testing process of Geant4, with some peculiarities specific to the physics domain addressed and to the project itself.

According to the guidelines of Geant4 testing process, the code is subject to unit tests at the level of each individual class developed, and to integration and system tests, which verify the correct behavior of the software within a full Geant4 
application. Regular Geant4 tests also include memory leak checking and monitoring of compliance with coding guidelines and software standards.

A specific feature of the test process of the project is the definition of an evaluation mission associated to every development cycle: it identifies the objectives and deliverables of the testing process appropriate to each stage of the project. Other aspects of the testing process are specific to the physics domain of the software, such as the validation against experimental data; an overview of their results at the current stage of the project is documented in section $\mathrm{V}$.

\section{SOFTWARE DESIGN FEATURES}

The Geant4-DNA software design reflects the domain decomposition derived from the analysis process. The relevant domain entities to be modeled are physics processes; each process encompasses the calculation of its cross section and the generation of a final state describing the interaction products. All particle collisions are treated on a single eventby-event basis; that is, in terms of Geant4 design concepts, all Geant4-DNA processes are discrete. A process can be pertinent to one or more particle types.

The design of the Geant4-DNA physics processes exploits the features of Geant 4 architecture. Thanks to the object oriented technology it adopts, Geant4 physics is open to extension, without affecting any of the existing Geant4 kernel or user application code. Physics processes are handled transparently by Geant4 tracking through the interface of an abstract base class (G4VProcess); a specialization of this class (G4VDiscreteProcess) acts as a base class for processes describing discrete interaction. To be compliant with the existing design of Geant4 physics, the Geant4-DNA processes are subclasses of G4VDiscreteProcess.

The Geant4-DNA physics processes adopt a policy-based class design [10]. The usage of this design technique represents an innovative design approach in Geant4.

A policy-based design assembles classes with complex functionality out of simpler classes, each one responsible of a single behavioral or structural aspect. Policies define a class interface or a class template interface; they are more loosely defined than conventional abstract interfaces (i.e. classes consisting of pure virtual functions), as they are syntax oriented rather than signature oriented. A policy-based design is highly customizable; this feature is especially important in a software system associated to a complex scientific domain, where a variety of physics models have been proposed in literature and further significant evolution is expected.

A policy-based design can also provide advantages in terms of the software performance with respect to more conventional techniques to handle interchangeable models, such as, for instance, the encapsulation of algorithms in a Strategy Pattern [11]: in fact, policies are compile-time bound, since templates generate the code at compile time based on the types provided by the user.

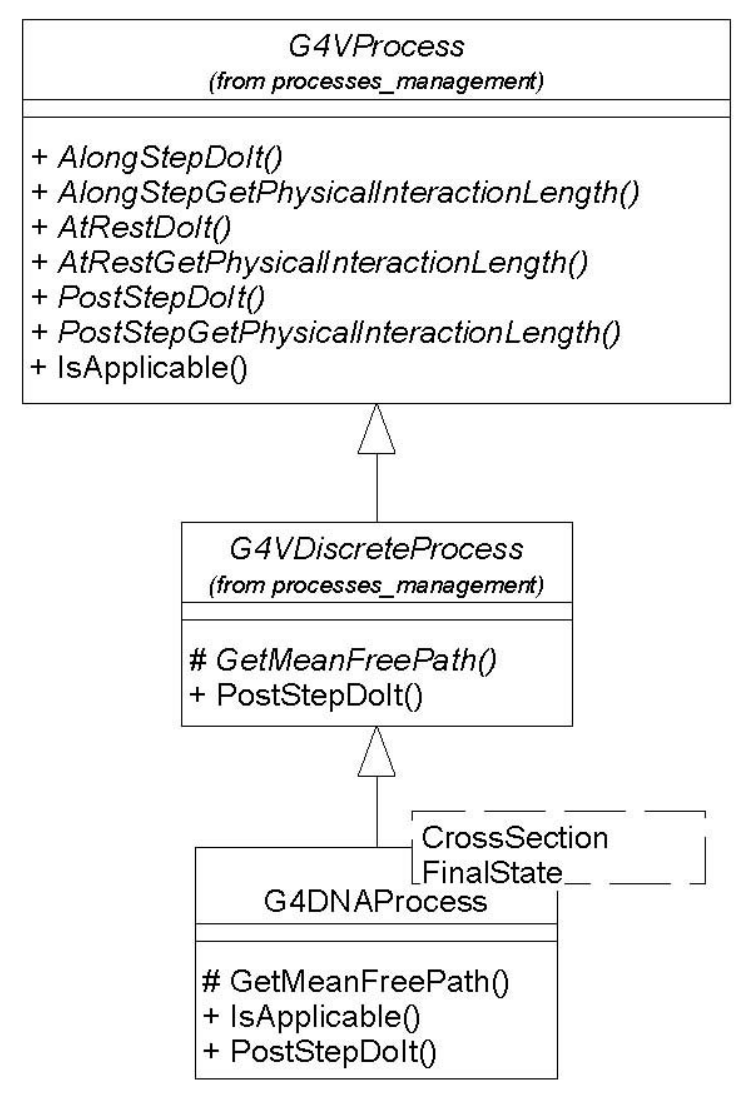

Fig. 1 The design of Geant4-DNA processes: G4DNAProcess is a parameterized class; its parameters are CrossSection and FinalState, which represent policy classes.

A generic Geant4-DNA physics process is characterized by two policies: the Cross Section policy and the Final State policy. The two policies identified to characterize a process are orthogonal (in terms of software design): that is, there is no dependency between either of them for a given process. The orthogonal decomposition of the host process class into policies is a key issue for a clean design.

The essential features of the Geant4-DNA physics design are shown in Fig. 1: a single parameterized class (G4DNAProcess), which inherits from G4VDiscreteProcess, defines the family of classes representing the Geant4DNA physics processes; it acts as a host class for the policies associated to its formal parameters: CrossSection and FinalState, respectively responsible for the calculation of the cross sections of a physics process and the generation of the products resulting from the interaction. This design allows customizing a single process class through multiple implementations of physics models: a specific physics process is configured by binding the formal parameters to policy classes implementing its cross section or final state model, as shown in

Fig. 2.

The versatility of the policy-based class design provides a common solution to various requirements typical of physics modeling: the same mechanism applies to describing different physics processes, alternative models for the same process, or the articulation of a physics process through complementary models (for instance, covering different energy ranges).

Alternative models for the same physics process can be configured by instantiating different policy classes for its cross section or final state: provided the cross section or final state 
classes supply an implementation of the respective policies, any models are interchangeable in the process configuration. Alternative models can even be supplied by a Geant4 user, for instance through experimental cross section measurements.

Complementary models, describing the cross sections or final state distributions over different energy ranges or target materials, can be aggregated into composite policy classes out of simple ones: an original design mechanism, which resembles the Composite [11] design pattern, has been devised for this purpose in the Geant4-DNA project. The management of composite models - both cross section and final state ones also adopts a policy-based class design: a PhysicsModel policy is defined, which concerns the energy boundaries of the validity of a model. The CrossSection and FinalState policies can be combined with the PhysicsModel policy in classes exhibiting multiple behaviors; this design solution avoids the drawbacks of multiple inheritance, while providing the software system a further level of versatility.

The design of the Geant4-DNA package has successfully explored modern design techniques, which are suitable to be applied to other Geant4 packages too. In this respect, it represents a prototype for a further design iteration extended to the whole Geant4 Low Energy Electromagnetic package.

The policies defined in the Geant4-DNA physics design are documented in Fig. 3.

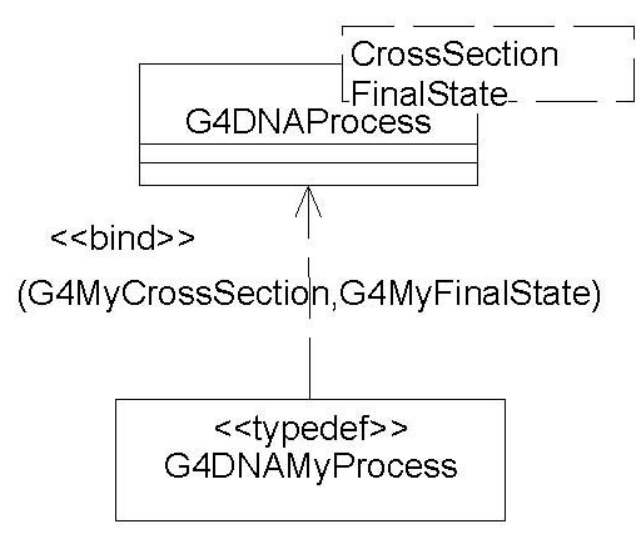

Fig. 2 An example of a template specialization : MyProcess is a specialization of G4DNAProcess.

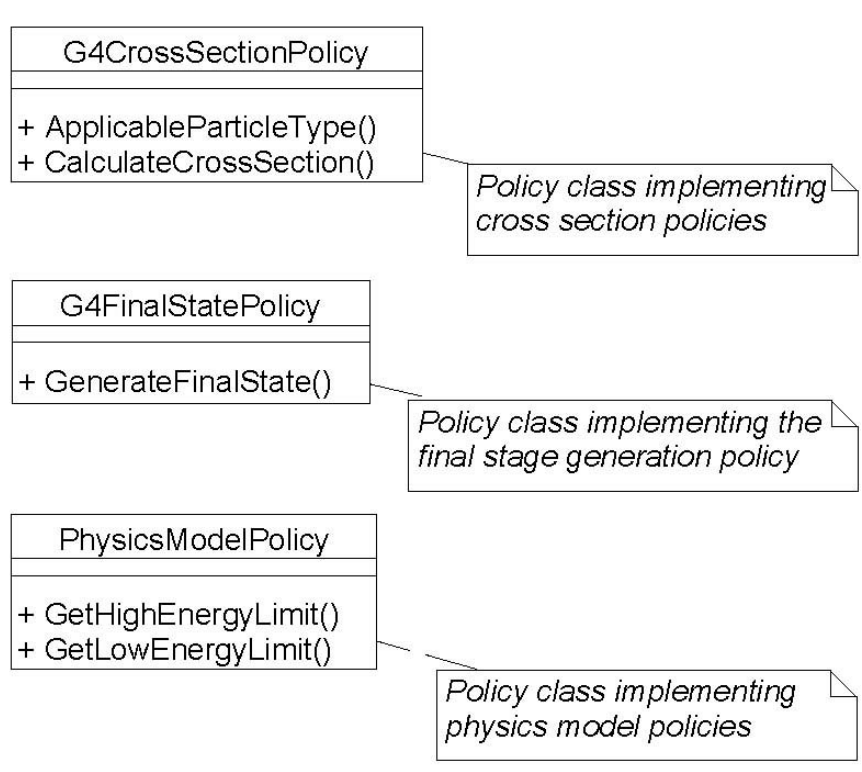

Fig. 3. Policy classes in the design of the Geant4-DNA physics.

\section{PHYSICS MODELS}

\section{A. Overview}

The Geant4-DNA physics processes cover the interactions of electrons, protons, neutral hydrogen, $\alpha$ particles and their charge states in liquid water down to the energy scale of a few electronvolts. They involve elastic scattering, excitation, charge change and ionisation. The processes currently implemented for each incident particle are listed in Table I.

All collisions are explicitly simulated as single-scattering interactions. This approach is suitable to micro- or nanodosimetry applications, and more in general to studies where the detailed structure of the energy deposit and of the secondary particle production associated to a track is essential; nevertheless, it results in a high computational demand, due to the large number of interactions that the particles involved experience before stopping.

Providing accurate inelastic cross-sections over the entire energy-loss range is one of the most important and difficult modeling tasks of a simulation system. Methods to model the hard events by means of an appropriate binary theory are well established: in this approach collisions are treated as binary processes, that is, either the target electrons are treated as free and at rest, or the influence of binding is accounted only roughly. However, accounting for the soft energy-loss collisions (10-100 eV) should involve the detailed knowledge of the electronic properties of the target, to ensure the correct treatment of energy loss occurrences of magnitude comparable to electronic binding energies: this is matter of considerable ongoing theoretical and computational effort.

The open design described in section III, suitable to the long term evolution of the software system and capable of providing a variety of model implementations, is the key issue to cope with the intrinsic modeling difficulties and the active theoretical research in this physics domain. 
The models described here correspond to the implementations released at the end of the second development cycle; they were chosen as the result of a survey of the theoretical and phenomenological developments documented in literature. The current set models implemented is not meant to be exhaustive; the initial selection will be expanded in future software development cycles, according to the incremental-iterative software process adopted.

TABLE I

GEANT4-DNA PHYSICS PROCESSES IMPLEMENTED IN THE GEANT4 TOOLKIT

\begin{tabular}{|l|l|}
\hline Incident particle & Processes \\
\hline Electron & $\begin{array}{l}\text { Elastic scattering } \\
\text { Excitation } \\
\text { Ionisation }\end{array}$ \\
\hline Proton & $\begin{array}{l}\text { Charge decrease } \\
\text { Excitation } \\
\text { Ionisation }\end{array}$ \\
\hline $\mathrm{H}$ & $\begin{array}{l}\text { Charge increase } \\
\text { Ionisation }\end{array}$ \\
\hline $\mathrm{He}++$ & $\begin{array}{l}\text { Charge decrease } \\
\text { Excitation } \\
\text { Ionisation }\end{array}$ \\
\hline $\mathrm{He}+$ & $\begin{array}{l}\text { Charge decrease } \\
\text { Charge increase } \\
\text { Excitation } \\
\text { Ionisation }\end{array}$ \\
\hline $\mathrm{He}$ & $\begin{array}{l}\text { Charge increase } \\
\text { Excitation } \\
\text { Ionisation }\end{array}$ \\
\hline
\end{tabular}

\section{B. Processes for the interactions of electrons}

Electron interactions involve elastic scattering, excitation and ionization. The description of these processes is of fundamental importance in the simulation of radiation effects to biological systems, since secondary electrons resulting from the interactions of other primary particles contribute significantly to the energy deposit.

1) Elastic scattering

The current implementation of this process provides one model for the calculation of the total cross section and two complementary models for the final state generation.

The angular distribution of the scattered electron is derived from two models of the differential cross section, respectively valid in the energy range from $3.5 \mathrm{eV}$ to $200 \mathrm{eV}$ and above 200 $\mathrm{eV}$. The lower energy model is based on a semi-empirical calculation [12], while the higher energy one implements the screened [13]-[14] Rutherford differential cross section [15] including the correction parameter documented in [16].

The total cross section is calculated from the integrated screened Rutherford formula; this approach has been demonstrated to be valid down to the scale of a few electronvolts [17].

2) Excitation

This process is described in the energy range from $7.5 \mathrm{eV}$ to
$10 \mathrm{keV}$. It takes into account five excitation levels of the water molecule: $\mathrm{A}^{1} \mathrm{~B}_{1}, \mathrm{~B}^{1} \mathrm{~A}_{1}$, Ryd $\mathrm{A}+\mathrm{B}$, Ryd $\mathrm{C}+\mathrm{D}$ and diffuse bands [18].

The total cross sections for excitation for each discrete transition are calculated analytically taking into account a lowenergy adjusting function [19].

The direction of the incident electron is left unchanged in the final state, since deflections associated to this process would be negligible [20] with respect to the effects related to elastic scattering.

3) Ionisation

The ionisation by incident electrons is also described in the same energy range as the excitation.

It adopts a semi-empirical model [18], which is based on the dielectric formalism for the valence shells responsible for condensed-phase effects, and on the binary encounter approximation for the K-shell.

To improve the software performance, the individual shell total cross sections [19] are computed from the interpolation of bi-dimensional pre-calculated tables over energy and momentum transfer.

The angular distribution of the outgoing electron is calculated from kinematical constraints.

\section{Processes for the interaction of protons}

The interactions of protons concern excitation, ionization and charge transfer. and neutral hydrogen are described from $100 \mathrm{eV}$ up to $10 \mathrm{MeV}$.

\section{1) Excitation}

Two alternative approaches are used to calculate the cross sections.

The first one [21] takes into account the five excitation levels of the water molecule described. It consists of two complementary models for the calculation of the cross sections: a semi-empirical one, originally based on [22], covers the energy range from $10 \mathrm{eV}$ to $500 \mathrm{keV}$, while a method based on the Born theory is used from $500 \mathrm{keV}$ up to $10 \mathrm{MeV}$. The individual excitation cross sections are computed from the interpolation of mono-dimensional tables computed by the authors of the model [23].

The second approach [24]-[25] takes into account eight electronic excitation states of the water molecule and it can be applied in the energy range between $300 \mathrm{keV}$ and $10 \mathrm{MeV}$; it is empirically based on electron impact data following the appropriate velocity scaling for protons. Pre-calculated tables provided by the authors [26] are used in the software implementation.

The incident proton direction is not modified in the generation of the final state.

\section{2) Ionisation}

The calculation of the cross sections is based on two models: a semi-empirical analytical approach described in [27]-[28] in the energy range between $100 \mathrm{eV}$ and $500 \mathrm{keV}$ of this process is parameterized in the $100 \mathrm{eV}-10 \mathrm{MeV}$ region from, and a model based on the Born theory [21] for energies 
above $500 \mathrm{keV}$ up to $10 \mathrm{MeV}$. The software implementation of the latter interpolates bi-dimensional pre-calculated tables depending on the energy and momentum transfers [23].

The generation of the energy spectrum of the secondary electrons is based on [29].

\section{3) Charge transfer}

The calculation of the cross section adopts an analytical formula as in [21]; the model is applicable from $100 \mathrm{eV}$ up to $10 \mathrm{MeV}$.

\section{Processes for the interaction of hydrogen}

The ionization and charge increase (stripping) of neutral hydrogen are described from about $100 \mathrm{eV}$ up to $10 \mathrm{MeV}$. The

The ionization differential cross sections are modeled as in [21] by applying a scaling factor with respect to the proton ionisation differential cross section. The total cross section is evaluated accordingly.

The cross section for charge increase (stripping) is modeled according to the analytical formula in [21].

\section{E. Processes for the interaction of helium ions}

These processes are modeled by applying an effective charge scaling [30], which takes into account the particle's electron screening, to the corresponding models for protons.

\section{PHYSICS TESTS}

The test of the simulation code encompasses processes peculiar to the domain it addresses, i.e. physics modeling: the verification that the software implementation reproduces the theoretical models correctly and the validation of the software against experimental data. The test mission of the second Geant4-DNA development was focused on verification; experimental validation will be the main object of a further dedicated development cycle.

\section{A. Verification of the physics models}

The compliance of the Geant4-DNA physics software with the underlying theoretical models is verified in unit tests associated to each policy class. The results of the Geant4 simulation are compared against references derived from the theoretical sources of the models implemented, such as, for instance, tabulated values directly provided by the authors of the theoretical models. Two results of the verification process are shown in Fig. 4 and Fig. 5, respectively concerning the cross sections for electron elastic scattering and proton excitation. It is evident that the software reproduces the reference theoretical models correctly.

Fig. 6 shows the comparison of the Geant4-DNA cross section calculations for proton and hydrogen charge transfer against data in [31]-[35]. These experimental data were used to determine the parameters of the theoretical model underlying the software implementation; therefore, the comparison may not be considered a proper software validation.

A systematic study for the validation of the Geant4-DNA physics processes against experimental data will be the object of a following dedicated paper.

\section{CONCLUSIONS AND OUTLOOK}

A novel set of processes to describe the interactions of various incident particles with liquid water down to the electronvolt scale has been included in Geant4. Thanks to these novel developments, for the first time a general-purpose Monte Carlo code can address a physics domain relevant to microdosimetry in biological systems.

The sophisticated software technology adopted makes the Geant4-DNA subsystem open to easy extension and evolution; the initial set of physics models implemented in the first development cycle will be complemented by further ones in future Geant4 releases.

\section{ACKNOWLEDGMENT}

The authors are grateful to Michael Dingfelder and Dimitris Emfietzoglou for their theoretical support to the software developments described in this paper and for providing tabulated results of their calculations. They wish to thank Werner Friedland and Herwig G. Paretzke for fruitful discussions, Riccardo Capra for contributing to a preliminary code prototype, Gabriele Cosmo and Ian Mc Laren for support and suggestions in the system testing process.

\section{REFERENCES}

[1] S. Agostinelli, J. Allison, K. Amako, J. Apostolakis, H. Araujo, P. Arce, et al., "Geant4: a Simulation Toolkit", Nucl. Instrum. Meth. A, vol. 506, no. 3, pp. 250-303, 2003.

[2] J. Allison, K. Amako, J. Apostolakis, H. Araujo, P. Arce Dubois, M. Asai, et al., "Geant4 Developments and Applications", IEEE Trans. Nucl. Sci., vol. 53, no. 1, pp. 270-278, 2006.

[3] S. Chauvie, S. Guatelli, V. Ivanchenko, F. Longo, A. Mantero, B. Mascialino, et al., "Geant4 Low Energy Electromagnetic Physics", in Conf. Rec. 2004 IEEE Nuclear Science. Symposium, N33-165.

[4] S. Chauvie, G. Depaola, V. Ivanchenko, F. Longo, P. Nieminen, and M. G. Pia, "Geant4 Low Energy Electromagnetic Physics", in Proc. Computing in High Energy and Nuclear Physics, Beijing, China, 2001, pp. 337-340.

[5] I. Jacobson, J. Booch, and J. Rumbaugh, The Unified Software Development Process, 1st ed., Ed: Addison-Wesley, 1999.

[6] P. Kruchten, The Rational Unified Process: An introduction, 3rd ed., Ed: Addison-Wesley, 2003.

[7] S. Guatelli, B. Mascialino, L. Moneta, I. Papadopoulos, A. Pfeiffer, M. G. Pia, et al., "Experience with Software Process in Physics Projects", in Conf. Rec. 2004 IEEE Nuclear Science Symposium, N40-8.

[8] Rational Software Corporation, Assessing the Rational Unified Process againstISO/IEC15504-5: 2000.

[9] ISO/IEC Joint Technical Committee 1, "ISO/IEC DTR 15504 Part 5: An Assessment Model and Indicator Guidance", Ed: J. M. Simon.

[10] A. Alexandrescu, Modern C++Design, Ed.: Addison-Wesley, 2001.

[11] E. Gamma, R. Helm, R. Johnson, and J. Vlissides, Design Patterns: Ed.: Addison-Wesley, 1994.

[12] D. J. Brenner, and M. Zaider, "A computationally convenient parameterization of experimental angular distributions of low energy electrons elastically scattered off water vapour", Phys. Med. Biol., vol. 29, no. 4, pp. 443-447, 1983.

[13] G. Molière, Z. Naturf., vol. A 2, pp. 133, 1947.

[14] G. Molière, Z. Naturf, vol.. A 3, pp. 78, 1948.

[15] H. Bethe, Phys. Rev., vol. 89 pp. 1256, 1953.

[16] B. Grosswendt and E. Waibel, "Transport of low energy electrons in nitrogen and air", Nucl. Instrum. Meth., vol. 155, pp. 145-156, 1978. 
[17] B. Senger, S. Fall, and R. V. Rechenmann, "Semi-empirical expressions describing the elastic scattering of slow electrons by molecules", Rad. Prot. Dosim., vol. 31, pp. 37-42, 1990.

[18] D. Emfietzoglou, and M. Moscovitch, "Inelastic collision characteristics of electrons in liquid water", Nucl. Instrum. Meth. B, vol. 193, pp. 71-78, 2002.

[19] D. Emfietzoglou, K. Karava, G. Papamichael, and M. Moscovitch, "Monte Carlo simulation of the energy loss of low-energy electrons in liquid water", Phys. Med. Biol., vol. 48, pp. 2355-2371, 2003.

[20] D. Emfietzoglou, G. Papamichael, and M. Moscovitch, "An event-byevent computer simulation of interactions of energetic charged particles and all their secondary electrons in water", J. Phys. D: Appl. Phys., vol. 33, pp. 932-944, 2000.

[21] M. Dingfelder, M. Inokuti, and H. G. Paretzke, "Inelastic-collision cross sections of liquid water for interactions of energetic protons", Rad. Phys. Chem., vol. 59, pp. 255-275, 2000.

[22] J. H. Miller and A. E. S. Green, "Proton Energy Degradation in Water Vapor", Rad. Res., vol. 54, pp. 343-363, 1973.

[23] M. Dingfelder, Department of Physics, East Carolina University, Greenville, USA, private communication.

[24] D. Emfietzoglou, G. Papamichael, K. Kostarelos, anad M. Moscovitch, "A Monte Carlo track structure code for electrons $(\sim 10 \mathrm{eV}-10 \mathrm{keV})$ and protons $(\sim 0.3-10 \mathrm{MeV})$ in water: partitioning of energy and collision events", Phys. Med. Biol., vol. 45, pp. 3171-3194, 2000.

[25] D. Emfietzoglou, K. Karava, G. Papamichael, M. Moscovitch, "MonteCarlo calculations of radial dose and restricted-LET for protons in water", Radiat. Prot. Dosim., vol. 110, pp. 871-879, 2004.

[26] D. Emfietzoglou, Department of Medical Physics, University of Ioannina Medical School, Ioannina, Greece, private communication.

[27] M. E. Rudd, T. V. Goffe, R. D. DuBois, L. H. Toburen, "Cross sections for ionisation of water vapor by $7-4000 \mathrm{keV}$ protons", Phys. Rev. A, vol. 31, pp. 492-494, 1985.

[28] M. E. Rudd, Y.-K. Kim, D. H. Madison, and. J. W. Gallagher, "Electron production in proton collisions: total cross sections", Rev. Mod. Phys. Vol. 57, no. 4, pp. 965-994, Oct. 1985.

[29] M. E. Rudd, "User-firendly model for the energy distribution of electrons from proton or electron collisions", Nucl. Tracks Rad. Meas., vol 16, no. 2-3, pp. 213-218, 1989.

[30] M. Dingfelder, H. G. Paretzke, and L. H. Toburen, "An effective charge scaling model for ionization of partially dressed helium ions with liquid water", in Proc. of Monte Carlo 2005, Chattanooga, Tennessee, 2005.

[31] B. G. Lindsay, D. R. Sieglaff, K. A. Smith, and R. F. Stebbings, "Charge transfer of 0.5-, 1.5-, and 5-keV protons with $\mathrm{H}_{2} \mathrm{O}$ : absolute differential and integral cross sections", Phys. Rev. A, vol. 55, no. 5, pp. 3945-3946, 1997.

[32] K. H. Berkner, R. V. Pyle, and J. W. Stearns, "Cross sections for electron capture by 0.3 to $70 \mathrm{keV}$ deuterons in $\mathrm{H}_{2}, \mathrm{H}_{2} \mathrm{O}, \mathrm{CO}, \mathrm{CH}_{4}$, and $\mathrm{C}_{8} \mathrm{~F}_{16}$ gases", Nucl. Fus., vol. 10, pp. 145-149, 1970.

[33] R. Dagnac, D. Blanc, and D. Molina, "A study on the collision of hydrogen ions $\mathrm{H}_{1}{ }^{+}, \mathrm{H}_{2}{ }^{+}$and $\mathrm{H}_{3}{ }^{+}$with a water-vapour target", J. Phys. B: Atom. Molec. Phys., vol. 3, pp.1239-1251, 1970.

[34] L. H. Toburen, M. Y. Nakai, and R. A. Langley, "Measurement of highenergy charge transfer cross sections for incident protons and atomic hydrogen in various gases", Phys. Rev., vol. 171, no. 1, pp. 114-122, 1968.

[35] P. G. Cable, Ph. D. thesis, University of Maryland, 1967.

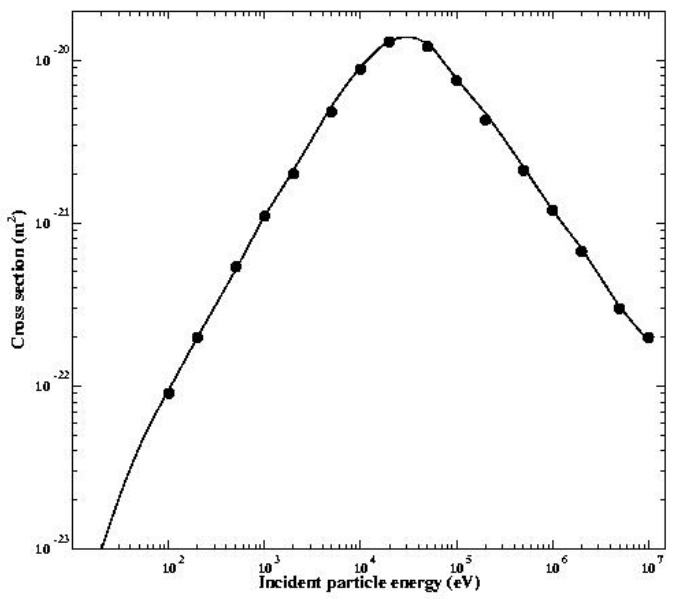

Fig. 4 Verification of the Geant4-DNA implementation of the cross sections for proton excitation; the black dots represent Geant4 simulation results, the solid line represents the theoretical model.

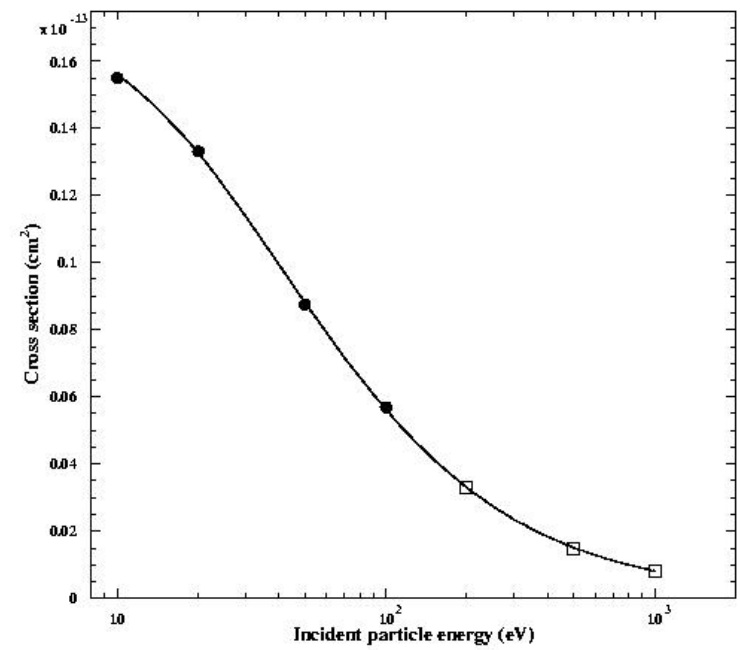

Fig. 5 Verification of the Geant4-DNA implementation of the cross sections for electron elastic scattering; the black dots represent Geant4 simulation results, the solid line represents the theoretical calculations [25].

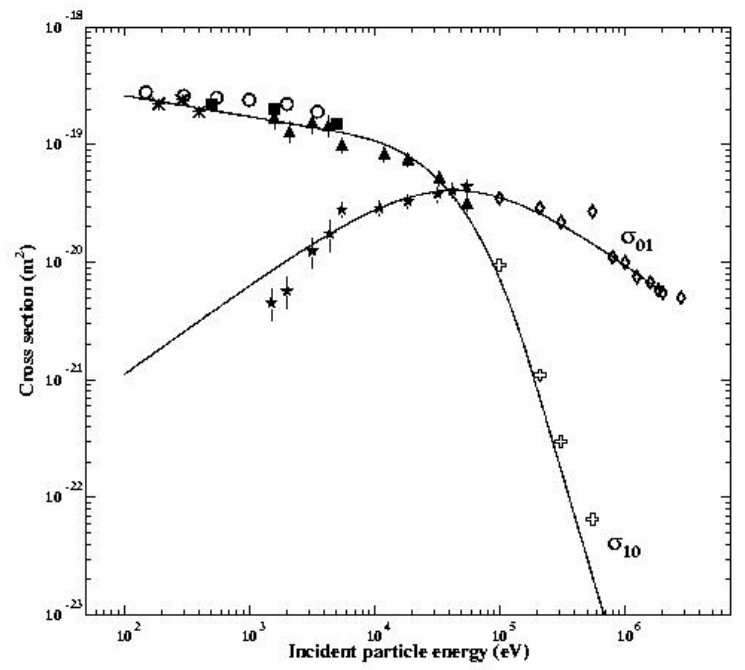

Fig. 6 Comparison of Geant4-DNA implementation of proton $\left(\sigma_{10}\right)$ and hydrogen $\left(\sigma_{01}\right)$ charge transfer against experimental measurements; the experimental data are from [31] (squares), [32] (circles), [33] (black stars), [33] (up triangles), [34] (diamonds), [34] (white crosses), and [35] (crosses). 